Pontifícia Universidade Católica $_{\text {da }}$

DO RIO DE JANEIRO

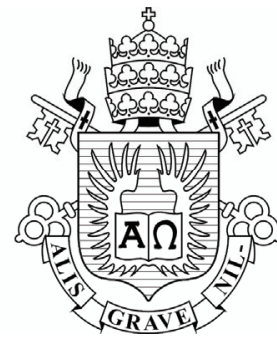

Thiago Souza Mendes Guimarães

\begin{abstract}
Apoio à Síntese de Modelos Estruturais de Software Orientado a Objetos Utilizando Algoritmos Genéticos Co-Evolucionários
\end{abstract}

Dissertação de Mestrado

Dissertação apresentada ao Programa de Pós-Graduação em Engenharia Elétrica da PUC-Rio como requisito parcial para obtenção do grau de Mestre em Engenharia Elétrica

Marco Aurélio C. Pacheco

Orientador

Departamento de Engenharia Elétrica - PUC-Rio 


$$
\text { Pontifícia } U_{\text {niversidade }} \text { Cé Rio de Janeica }_{\text {do }}
$$

Thiago Souza Mendes Guimarães

\section{Apoio à Síntese de Modelos Estruturais de Software Orientado a Objetos Utilizando Algoritmos Genéticos Co-Evolucionários}

Dissertação apresentada como requisito parcial para obtenção do grau de Mestre pelo Programa de PósGraduação em Engenharia Elétrica do Departamento de Engenharia Elétrica do Centro Técnico Científico da PUCRio. Aprovada pela Comissão Examinadora abaixo assinada.

Prof. Marco Aurélio Cavalcanti Pacheco

Orientador

Departamento de Engenharia Elétrica - PUC-Rio

Prof. Marley Maria Bernardes Rebuzzi Vellasco Departamento de Engenharia Elétrica - PUC-Rio

Prof. Carlos José Pereira de Lucena Departamento de Informática - PUC-Rio

Prof. Flávio Joaquim de Souza UERJ

Profa. Karla Tereza Figueiredo Leite UERJ

Prof. Carlos Roberto Hall Barbosa Instituto Tecnológico da PUC-Rio - ITUC

Prof. José Eugenio Leal Coordenador Setorial do Centro

Técnico Científico - PUC-Rio

Rio de Janeiro, 07 de abril de 2005 
Todos os direitos reservados. É proibida a reprodução total ou parcial do trabalho sem autorização do autor, do orientador e da universidade.

Thiago Souza Mendes Guimarães

Graduou-se em Bacharelado em Informática pela UFRJ em 2003

Ficha catalográfica

Guimarães, Thiago Souza Mendes

Apoio à síntese de modelos estruturais de software orientado a objetos utilizando algoritmos genéticos coevolucionários / Thiago Souza Mendes Guimarães ; orientador: Marco Aurélio C. Pacheco. - Rio de Janeiro : PUC-Rio, Departamento de Engenharia Elétrica, 2005.

127 f. il. ; $30 \mathrm{~cm}$

Dissertação (mestrado) - Pontifícia Universidade Católica do Rio de Janeiro, Departamento de Engenharia Elétrica .

Inclui bibliografia

1. Engenharia Elétrica - Teses. 2. Algoritmos genéticos. 3. Engenharia de software. 4. Co-evolução. 5. Qualidade de software. 6. Métricas de qualidade. I. Souza, Reinaldo Castro. II. Pontifícia Universidade Católica do Rio de Janeiro. Departamento de Engenharia Elétrica. III. Título.

CDD: 621.3 


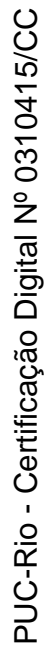

Para meus pais, Luiz Alberto e Glaucia. 


\section{Agradecimentos}

À CAPES e à PUC-Rio, pelos auxílios concedidos, sem os quais este trabalho não poderia ter sido realizado.

Ao meu orientador, Prof. Dr. Marco Aurélio C. Pacheco, pelo estímulo e parceria na realização deste trabalho.

Aos meus pais, pela educação e apoio.

Aos meus colegas da PUC-Rio e do ICA: André Abs da Cruz, Carlos Hall, Juan Lazo, Karla Figueiredo, Luciana Falleti, Yvan Tupac.

Aos meus familiares e amigos que de uma forma ou de outra me estimularam e ajudaram. 


\section{Resumo}

Guimarães, Thiago Souza Mendes . Apoio à Síntese de Modelos Estruturais de Software Orientado a Objetos Utilizando Algoritmos Genéticos Co-Evolucionários. Rio de Janeiro, 2005. 127p. Dissertação de Mestrado - Departamento de Engenharia Elétrica, Pontifícia Universidade Católica do Rio de Janeiro.

Esta dissertação investiga o uso de Algoritmos Genéticos Co-evolucionários na automatização do processo de desenvolvimento de Sistemas de Software Orientados a Objetos. A qualidade final do software depende principalmente da qualidade da modelagem desenvolvida para o mesmo. Durante a fase de modelagem, diversos modelos são desenvolvidos antecipando diversas visões do produto final, e possibilitando a avaliação do software antes mesmo que ele seja implementado. A síntese de um modelo de software pode, portanto, ser vista como um problema de otimização onde se busca uma melhor configuração entre os elementos contemplados pelo paradigma de orientação a objetos, como classes, métodos e atributos, que atenda a critérios de qualidade de design. O objetivo do trabalho foi estudar uma forma de sintetizar modelagens de maior qualidade através da evolução por Algoritmos Genéticos Co-evolucionários. Para avaliar a modelagem do software, foram investigadas métricas de qualidade de software tais como: Reutilização, Flexibilidade, Inteligibilidade, Funcionalidade, Extensibilidade e Efetividade. Essas métricas foram aplicadas na função de avaliação, que por sua vez, foi definida objetivando a síntese de uma modelagem de software orientado a objetos com uma maior qualidade. Neste problema, deseja-se contemplar mais de um objetivo ao mesmo tempo. Para isso, foi utilizada a técnica de Pareto para problemas multi-objetivos.

Os resultados obtidos foram comparados com modelagens produzidas por especialistas e as suas características analisadas. O desempenho do AG no processo de otimização foi comparado com o da busca aleatória e, em todos os casos, os resultados obtidos pelo modelo foram sempre superiores.

\section{Palavras-chave}

Algoritmos Genéticos; Co-evolução; Engenharia de Software; Qualidade de Software; Métricas de Qualidade 


\section{Abstract}

Guimarães, Thiago Souza Mendes . Support to the Synthesis of Structural Models of Object-Oriented Software Using Co-Evolutionary Genetic Algorithms. Rio de Janeiro, 2005. 127p. Dissertação de Mestrado Departamento de Engenharia Elétrica, Pontifícia Universidade Católica do Rio de Janeiro.

This work investigates the use of Co-evolutionary Genetic Algorithms in the automation of the development process of object-oriented software systems. The software final quality depends mainly on the design quality developed for the same. During the design phase, different models are developed anticipating various visions of the end product, thus making possible the software evaluation before it is implemented. The synthesis of a software model can, therefore, be seen as an optimization problem where it seeks a better configuration between the contemplated elements for the object-oriented paradigm, as classes, methods and attributes, which follows the quality design criteria. The work goal was to study a way to synthesize designs of better quality through its evolution by Coevolutionary Genetic Algorithms. In order to assess the software quality, it was also investigated software quality metrics, such as: Reusability, Flexibility, Understandability, Functionality, Extensibility and Effectiveness. These metrics were applied in an evaluation function that, in turn, was defined aiming at the object-oriented design synthesis with a better quality. In this problem, it is desired to contemplate more than one objective at a time. For this, the Pareto technique for multi-objective problems was used.

The results were compared with designs produced by specialists and its characteristics analyzed. The GA performance in the optimization process was compared with the exhaustive search and, in all cases, the model results were superior.

\section{Keywords}

Genetic Algorithms; Co-evolution; Software Engineering; Software Quality; Quality Metrics 


\section{Sumário}

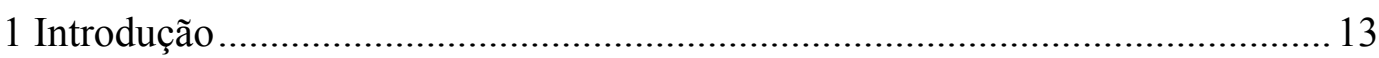

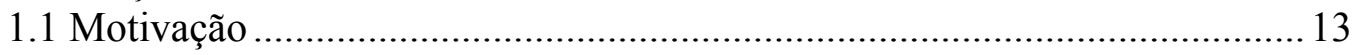

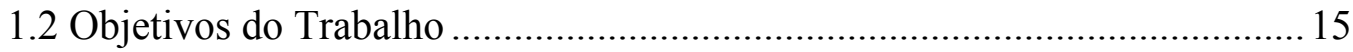

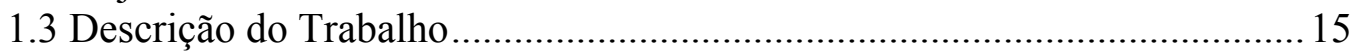

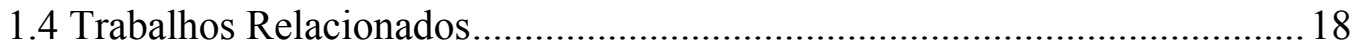

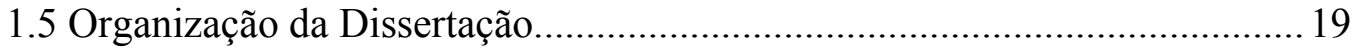

2 Engenharia de Software .............................................................................. 20

2.1 Design de Sistemas Orientados a Objetos .................................................... 20

2.2 Métricas de Qualidade de Software.............................................................2

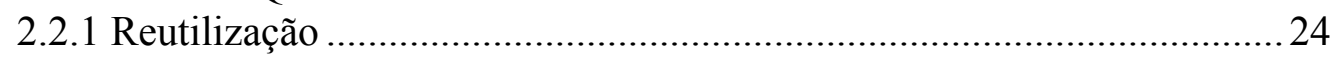

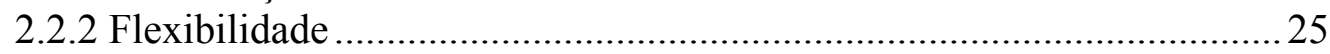

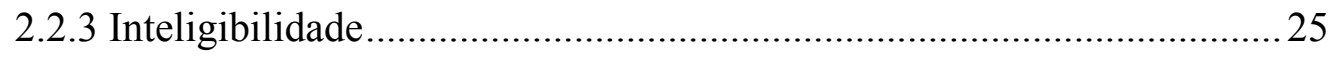

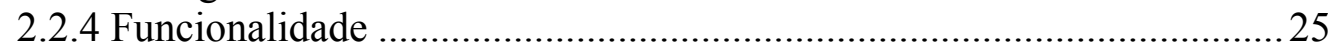

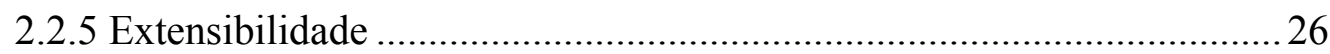

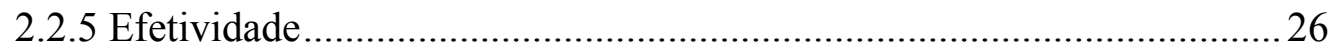

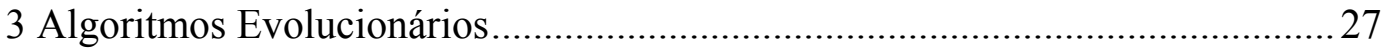

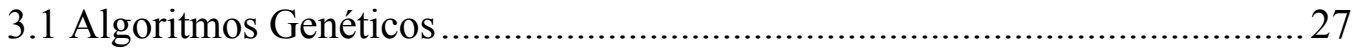

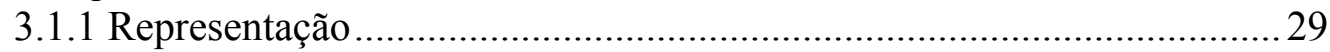

3.1.2 Codificação e Decodificação ................................................................29

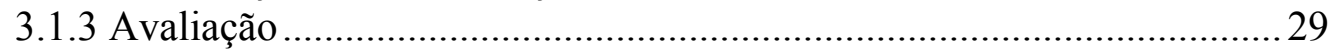

3.1.4 Operadores Genéticos............................................................................. 30

3.1.5 Parâmetros da Evolução ......................................................................... 31

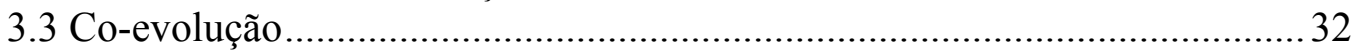

3.4 Estratégias de avaliação multi-objetivo............................................................ 37

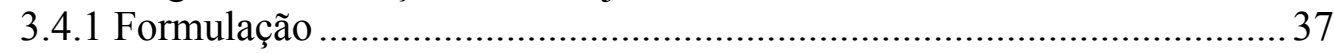

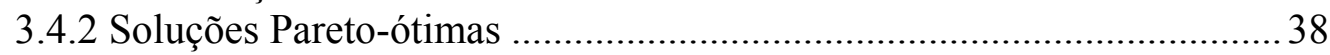

4 Sistema de Evolução de Designs Baseada em Qualidade de Software Orientado

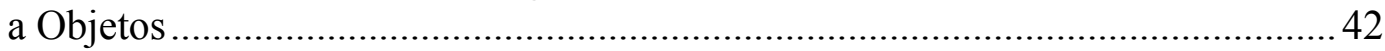

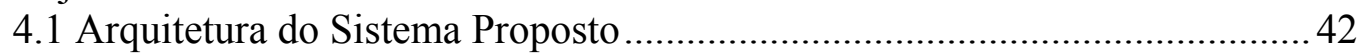

4.2 Síntese de Design por Algoritmos Genéticos Co-Evolucionários.................. 45

4.2.1 Representação das Espécies.................................................................... 46

4.2.2 Operadores Genéticos....................................................................... 51

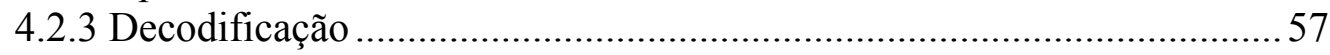

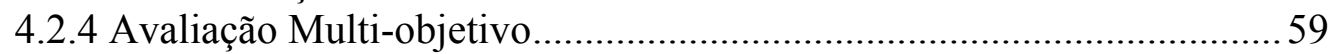

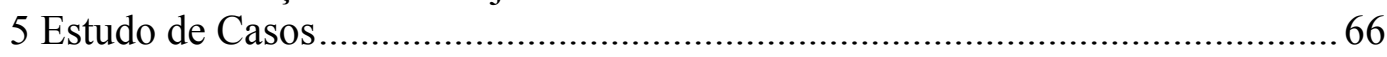

5.1 Estudo de Caso 1: Modelagens simples ........................................................ 68

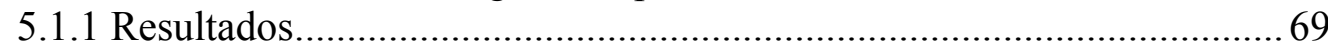

5.2 Estudo de Caso 2: Controle de Elevador ...................................................... 76

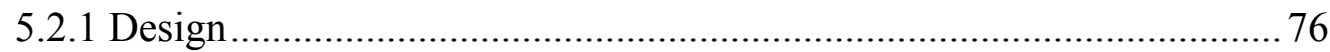

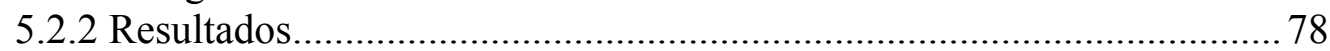

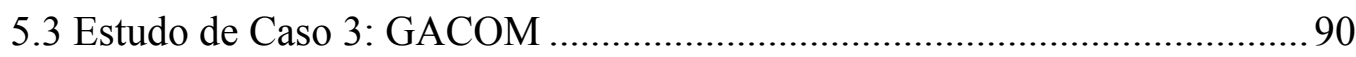

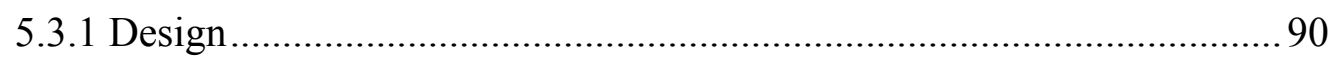

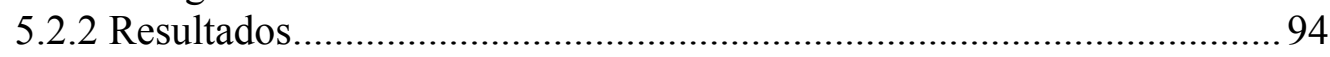

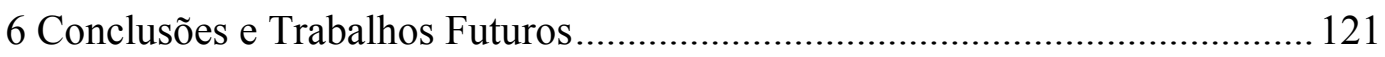

7 Referências Bibliográficas............................................................................ 124 


\section{Lista de Figuras}

Figura 1 - Descrição do processo de automatização do desenvolvimento do software

Figura 2 - Exemplo da relação de Herança 21

Figura 3 - Exemplo da relação de Dependência 22

Figura 4 - Procedimento básico do algoritmo genético 28

Figura 5 - Cruzamento de um ponto $\quad 30$

Figura 6 - Mutação 31

Figura 7 - Gráfico da equação $\quad 34$

Figura 8 - Gráfico para x igual a $40 \quad 34$

Figura 9- Gráfico para x igual a $-40 \quad 35$

Figura 10 - Modelo co-evolucionário genérico 36

Figura 11 - Exemplo que ilustra várias opções de compra de carro (1-5), considerando o seu custo e conforto

Figura 12 - Distribuição de Soluções na Fronteira de Pareto 41

Figura 13 - Descrição do processo de síntese da modelagem 42

Figura 14 - Visão geral do Modelo Genético com as quatro espécies 47

Figura 15 - Diagrama de classes simplificado $\quad 47$

Figura 16 - Representação gráfica das dependências do cromossomo da Tabela 11

Figura 17 - Exemplo do ONEPOINT crossover em duas dimensões $\quad 56$

$\begin{array}{ll}\text { Figura } 18 \text { - Modelagem sintetizada } & 59\end{array}$

$\begin{array}{ll}\text { Figura } 19 \text { - Modelagem sintetizada } & 70\end{array}$

Figura 20 - Modelagem sintetizada otimizando atributo de Reutilização $\quad 71$

Figura 21 - Modelagem sintetizada otimizando atributo de Flexibilidade $\quad 72$

Figura 22 - Modelagem sintetizada otimizando atributo de Inteligibilidade $\quad 72$

Figura 23 - Modelagem sintetizada otimizando atributo de Funcionalidade $\quad 73$

Figura 24 - Modelagem sintetizada otimizando atributo de Extensibilidade $\quad 73$

Figura 25 - Modelagem sintetizada otimizando atributo de Efetividade $\quad 74$

Figura 26 - Modelagem elaborada por um especialista do problema do elevador 77

Figura 27 - Gráfico de desempenho do AG para o objetivo Reutilização $\quad 78$

Figura 28 - Gráfico de desempenho do AG para o objetivo Flexibilidade $\quad 79$

Figura 29 - Gráfico de desempenho do AG para o objetivo Inteligibilidade 79

Figura 30 - Gráfico de desempenho do AG para o objetivo Funcionalidade 79

Figura 31 - Gráfico de desempenho do AG para o objetivo Extensibilidade $\quad 80$

Figura 32 - Gráfico de desempenho do AG para o objetivo Efetividade $\quad 80$

Figura 33 - Gráfico de evolução da métrica DSC $\quad 81$

Figura 34 - Gráfico de evolução da métrica NOH $\quad 81$

Figura 35 - Gráfico de evolução da métrica ANA $\quad 82$

Figura 36 - Gráfico de evolução da métrica MOA $\quad 82$

Figura 37 - Gráfico de evolução da métrica DCC $\quad 82$

Figura 38 - Gráfico de evolução da métrica CAM $\quad 83$

Figura 39 - Gráfico de evolução da métrica MFA $\quad 83$

Figura 40 - Gráfico de evolução da métrica CIS $\quad 83$

Figura 41 - Gráfico da otimização independente do atributo Reutilização $\quad 85$

Figura 42 - Gráfico da otimização independente do atributo Flexibilidade $\quad 85$

Figura 43 - Gráfico da otimização independente do atributo Inteligibilidade 85

Figura 44 - Gráfico da otimização independente do atributo Funcionalidade 86 
Figura 45 - Gráfico da otimização independente do atributo Extensibilidade $\quad 86$

Figura 46 - Gráfico da otimização independente do atributo Efetividade 86

Figura 47 - Modelagem sintetizada na otimização de todos os atributos de qualidade

Figura 48 - Modelagem sintetizada na otimização da Inteligibilidade $\quad 89$

Figura 49 - Otimização da Inteligibilidade e Flexibilidade usando semente inicial

Figura 50 - Módulo do processo de evolução do GACOM

Figura 51 - Comparativo da evolução do AG X Busca aleatória. AG 42,6\%

melhor
Figura 52 - Comparativo da evolução do AG X Busca aleatória. AG 3,9\% melhor

Figura 53 - Comparativo da evolução do AG X Busca aleatória. AG 21\% pior 95

Figura 54 - Comparativo da evolução do AG X Busca aleatória. AG 19,25\% melhor

Figura 55 - Comparativo da evolução do AG X Busca aleatória. AG 10,7\% melhor

Figura 56 - Comparativo da evolução do AG X Busca aleatória. AG 9,9\% melhor

Figura 57 - Modelagem sintetizada do GACOM, para todos os objetivos

Figura 58 - Gráfico de desempenho para o atributo Reutilização 97

Figura 59 - Gráfico de monitoramento da métrica DSC durante a evolução 98

Figura 60 - Gráfico de monitoramento da métrica NOH durante a evolução 98

Figura 61 - Gráfico de monitoramento da métrica ANA durante a evolução 98

Figura 62 - Gráfico de monitoramento da métrica MOA durante a evolução 99

Figura 63 - Gráfico de monitoramento da métrica DCC durante a evolução 99

Figura 64 - Gráfico de monitoramento da métrica CAM durante a evolução 99

Figura 65 - Gráfico de monitoramento da métrica MFA durante a evolução 100

Figura 66 - Gráfico de monitoramento da métrica CIS durante a evolução $\quad 100$

Figura 67 - Gráfico de desempenho para o atributo Flexibilidade 101

Figura 68 - Gráfico de monitoramento da métrica DSC durante a evolução 101

Figura 69 - Gráfico de monitoramento da métrica NOH durante a evolução 101

Figura 70 - Gráfico de monitoramento da métrica ANA durante a evolução 102

Figura 71 - Gráfico de monitoramento da métrica MOA durante a evolução 102

Figura 72 - Gráfico de monitoramento da métrica DCC durante a evolução 102

Figura 73 - Gráfico de monitoramento da métrica CAM durante a evolução 103

Figura 74 - Gráfico de monitoramento da métrica MFA durante a evolução 103

Figura 75 - Gráfico de monitoramento da métrica CIS durante a evolução 103

Figura 76 - Gráfico de desempenho para o atributo Inteligibilidade $\quad 104$

Figura 77 - Gráfico de monitoramento da métrica DSC durante a evolução 104

Figura 78 - Gráfico de monitoramento da métrica NOH durante a evolução 105

Figura 79 - Gráfico de monitoramento da métrica ANA durante a evolução 105

Figura 80 - Gráfico de monitoramento da métrica MOA durante a evolução 105

Figura 81 - Gráfico de monitoramento da métrica DCC durante a evolução 106

Figura 82 - Gráfico de monitoramento da métrica CAM durante a evolução 106

Figura 83 - Gráfico de monitoramento da métrica MFA durante a evolução 106

Figura 84 - Gráfico de monitoramento da métrica CIS durante a evolução 107

Figura 85 - Gráfico de desempenho para o atributo Funcionalidade $\quad 107$

Figura 86 - Gráfico de monitoramento da métrica DSC durante a evolução 108

Figura 87 - Gráfico de monitoramento da métrica NOH durante a evolução 108 
Figura 88 - Gráfico de monitoramento da métrica ANA durante a evolução 108

Figura 89 - Gráfico de monitoramento da métrica MOA durante a evolução 109

Figura 90 - Gráfico de monitoramento da métrica DCC durante a evolução 109

Figura 91 - Gráfico de monitoramento da métrica CAM durante a evolução 109

Figura 92 - Gráfico de monitoramento da métrica MFA durante a evolução 110

Figura 93 - Gráfico de monitoramento da métrica CIS durante a evolução 110

Figura 94 - Gráfico de desempenho para o atributo Extensibilidade 111

Figura 95 - Gráfico de monitoramento da métrica DSC durante a evolução 111

Figura 96 - Gráfico de monitoramento da métrica NOH durante a evolução 111

Figura 97 - Gráfico de monitoramento da métrica ANA durante a evolução 112

Figura 98 - Gráfico de monitoramento da métrica MOA durante a evolução 112

Figura 99 - Gráfico de monitoramento da métrica DCC durante a evolução 112

Figura 100 - Gráfico de monitoramento da métrica CAM durante a evolução 113

Figura 101 - Gráfico de monitoramento da métrica MFA durante a evolução 113

Figura 102 - Gráfico de monitoramento da métrica CIS durante a evolução 113

Figura 103 - Gráfico de desempenho para o atributo Efetividade $\quad 114$

Figura 104 - Gráfico de monitoramento da métrica DSC durante a evolução 114

Figura 105 - Gráfico de monitoramento da métrica NOH durante a evolução 115

Figura 106 - Gráfico de monitoramento da métrica ANA durante a evolução 115

Figura 107 - Gráfico de monitoramento da métrica MOA durante a evolução 115

Figura 108 - Gráfico de monitoramento da métrica DCC durante a evolução 116

Figura 109 - Gráfico de monitoramento da métrica CAM durante a evolução 116

Figura 110 - Gráfico de monitoramento da métrica MFA durante a evolução 116

Figura 111 - Gráfico de monitoramento da métrica CIS durante a evolução 117

Figura 112 - Gráfico de evolução da métrica NOH separadamente. Métrica DSC

Figura 113 - Gráfico de evolução da métrica NOH separadamente. Métrica NOH

Figura 114 - Gráfico de evolução da métrica NOH separadamente. Métrica ANA

Figura 115 - Gráfico de evolução da métrica NOH separadamente. Métrica MOA

Figura 116 - Gráfico de evolução da métrica NOH separadamente. Métrica DCC

Figura 117 - Gráfico de evolução da métrica NOH separadamente. Métrica CAM

Figura 118 - Gráfico de evolução da métrica NOH separadamente. Métrica MFA

Figura 119 - Gráfico de evolução da métrica NOH separadamente. Métrica CIS 


\section{Lista de Tabelas}

Tabela 1 - Definição dos Atributos de Qualidade $\quad 24$

Tabela 2 - Especificação de Atributos $\quad 43$

Tabela 3 - Especificação de Métodos $\quad 43$

Tabela 4 - Especificação de Relações $\quad 43$

Tabela 5 - Exemplo de Especificação de Atributos 43

Tabela 6 - Exemplo de Especificação de Métodos 44

Tabela 7 - Exemplo de Especificação de Relações 44

Tabela 8 - Exemplo de cromossomo com máximo de 8 classes 48

Tabela 9 - Exemplo de máscara de ativação ativando 4 classes 48

Tabela 10 - Máscara representando uma matriz de incidência correspondente ao diagrama de classes da Figura $15 \quad 49$

Tabela 11 - Exemplo de cromossomo da espécie Dependências $\quad 50$

Tabela 12 - Exemplo de cromossomo da espécie Métodos, com MaxClasses $=6$

Tabela 13 - Exemplo de cromossomo da espécie Atributos, com MaxClasses $=6$

Tabela 14 - Definição das Propriedades de uma modelagem 60

Tabela 15 - Equações para inferência dos atributos de qualidade $\quad 60$

Tabela 16 - Descrição das métricas $\quad 61$

Tabela 17 - Relação das métricas com as propriedades da modelagem $\quad 61$

Tabela 18 - Relação das métricas com os componentes básicos da modelagem 62

Tabela 19 - Comparação dos atributos de qualidade das modelagens $\quad 87$

Tabela 20 - Comparação das modelagens 97 